\title{
Analgesic Contrast of Diverse Administrations of Botulinum Neurotoxin A (BoTN/A) Using Rat Neuropathic Pain Model
}

\author{
Qiong-Fang YANG ${ }^{1,3}, \mathrm{Li}-\mathrm{Zu} \mathrm{XIAO}^{2}$, Wei-Jiao XIE ${ }^{1,2, a, ~}{ }^{*}$, Yun-Hai \\ QIU $^{1}$
}

\author{
${ }^{1}$ Research Centre for Neural Engineering, Shenzhen Institutes of Advanced \\ Technology, Chinese Academy of Sciences, Shenzhen, China \\ ${ }^{2}$ TheDepartment of Pain, Shenzhen Sixth People's Hospital, Nanshan District, \\ Shenzhen, China \\ ${ }^{3}$ Shenzhen College of Advanced Technology, University of Chinese Academy of \\ Sciences, Shenzhen, China \\ aemail:wj.xie@siat.ac.cn \\ ${ }^{*}$ Corresponding author
}

Keywords: Botulinum Neurotoxin A, Neuropathic Pain, Administration.

\begin{abstract}
As a widely used clinical biomedicine for movement disorders and anti-wrinkle, in recent years, BoTN/A has emerged as a potential approach for treating refractory pain, especially migraine, diabetic pain, postherpetic neuralgia (PHN). In this study, we compared the analgesic effect of diverse administrations of Botulinum neurotoxin A (BoTN/A) on rat partial sciatic nerve ligation (PSNL) neuropathic pain model. Single intraplantar (i.pl.) and intrathecal (i.t.) injection of BoTN/A was applied after or before PSNL operation, respectively. The nociceptive behavioral tests showed single intraplantar injection after or before operation, limitedly blocked PSNL induced mechanical and thermal hypersensitivity, even at quite high dose (40U/kg). However, single BoTN/A intrathecal injection low dose (1U/kg) at 2 days previous operation, prevented the neuropathic pain initiation and facilitated functional recovery. These results indicated intrathecal pre-administration of BoTN/A has opportunity to be approved as preventive medication in clinical application.
\end{abstract}

\section{Introduction}

The family of Botulinum neurotoxins (BoTNs) comprises of seven distinct serotypes, termed A-G, characterized by cleaving proteins for docking or fusion of synaptic vesicles to the presynaptic membrane[1]. Botulinum neurotoxin A (BoTN/A) is the most prevalent used agent clinically, including numerous neuromuscular disorders and removal of facial wrinkles. After utilizing synaptic vesicle protein 2 (SV2) as receptor for entry into neurons [2], BoTN/A persists long-term catalytic activity to selectively cleave synaptosomal-associated protein of $25 \mathrm{kDa}$ (SNAP-25), resulting in blocking acetylcholine release at neuromuscular junction in peripheral motor neurons [3].This zinc-dependent proteolytic ability is well accepted as the mechanism underlying treating neuromuscular disorders and anti-wrinkles.

Recently, using BoTN/A as bio-therapeutic medicine to treat various types of pain, has raised more and more attentions[4]. The FDA had already approved BoTN/A for migraine relief, and European Federation of Neurological Societies had also recommended BoTN/A as Class II medicine for diabetic pain in the guidelines on the pharmacological treatment of neuropathic pain in 2010 [5]. The animal researches demonstrate BoTN/A blocks inflammatory or neuropathic pain by inhibiting the 
release of pain related neurotransmitters, such as glutamate [6], calcitonin gene-related peptide (CGRP) [7], Substance P [8]. Moreover, it has been reported that BoTN/A can be retrogradely transported from peripheral terminals to central nervous system [9]. Our previous clinical research has reported BoTN/A is able to relieve postherpetic neuralgia (PHN) significantly for monthly [10]. The existing theoretical basis falls short of explaining clinical application of BoTN/A intensively, and meanwhile the enlarged indication of BoTN/A is deserved to be explored. Herein, we used partial sciatic nerve ligation (PSNL) neuropathic pain model to inspect the different analgesic effects of diverse administrations of BoTN/A aiming at enlarging its application.

\section{Methods}

Animals

Male rats weighing 180-200g, provided by Experimental Animal Center of Guangdong Province were used. They were kept in a room with a temperature of $21 \pm$ $2^{\circ} \mathrm{C}$ with free access to standard laboratory diet and tap water. All procedures were approved by the Ethics Committee of SIAT and complied with the recommendations of the International Association for the Study of Pain.

\section{Partial Sciatic Nerve Ligation (PSNL)}

Partial ligation of the sciatic nerves of rats were performed under pentobarbital (50 $\mathrm{mg} / \mathrm{kg}$ i.p.) anesthesia, following the method of Malmberg and Basbaum [11]. Briefly, the common sciatic nerve of the right hind limb was exposed at high thigh level through a small incision and the dorsal half of the nerve thickness was tightly ligated with a silk suture.

\section{BoTN/A Administrations}

BoTN/A(Botox, Allergan, Inc., USA) solutions were prepared from stock solutions of 2000U/ml kept in liquid nitrogen, which were diluted to proper final concentration by sterilized $0.9 \%$ saline. For intraplantar (i.pl.) injection, rats were anesthetized by pentobarbital (50mg/kg i.p.). A $30 \mathrm{G}$ needle was inserted into the plantar surface of the right paw subcutaneously and 20ul BoTN/A was injected slowly over 30 seconds. For intrathecal (i.t.) injection, rats were anesthetized by pentobarbital $(50 \mathrm{mg} / \mathrm{kg}$ i.p.), and the back cortical layer was cut to expose vertebrae. A $28 \mathrm{G}$ needle was inserted between L5 and L6 vertebrae and 10ul BoTN/A was injected over 30 seconds. A significant tail flick could be observed during injection. The needle was kept in vertebrae interval 2 minutes after injection to avoid drug leaking.

\section{Behavioral Tests}

In the thermal paw withdrawal test, the nociception threshold was evaluated as the latency to withdraw a paw upon a thermal stimulus. Unanesthetized animals were placed in Plexiglas cages on top of a glass sheet and an adaptation period of $1 \mathrm{~h}$ was allowed. A thermal stimulator (IITC Inc., Woodland Hills, CA, USA) was positioned under the glass sheet and the focus of the projection bulb was aimed exactly at the middle of the plantar surface of the animal. A mirror attached to the stimulator permitted visualization of the plantar surface. A cut-off time of $30 \mathrm{sec}$ was set to prevent tissue damage. For the mechanical paw pressure test, rats were placed in a plexiglas chamber on a $6 \times 6 \mathrm{~mm}$ wire mesh grid floor and allowed to acclimatize for a period of $1 \mathrm{~h}$. A mechanical stimulus was then delivered to the middle of the plantar 
surface of the right-hind paw using a Transducer Indicator (Model 1601; IITC Inc., Woodland Hills, CA, USA). The amount of pressure that induced a flexor response was defined as the pain threshold.

\section{Time Course}

The nociceptive behavioral tests were performed before PSNL operation presented as day 0 , and then the tests were done post operation at day $3,5,7,15,20,26$.

\section{Statistical Analysis}

Data are presented as mean \pm SEM. Data were analyzed with Student's t-tests, $\mathrm{P}<0.05$ was set as the criterion for statistical significance.

\section{Results}

\section{PSNL Neuropathic Pain Model}

Partial sciatic nerve ligation model as a common used neuropathic pain model was chosen in our study. Compared with other models such as spinal nerve ligation (SNL), PSNL had minor injury to animals, and obvious autotomy was not discovered in whole experimental procedure. The data showed rats in PSNL groups resulted in stable long lasting mechanical allodynia and thermal hyperalgesia (Fig.1).
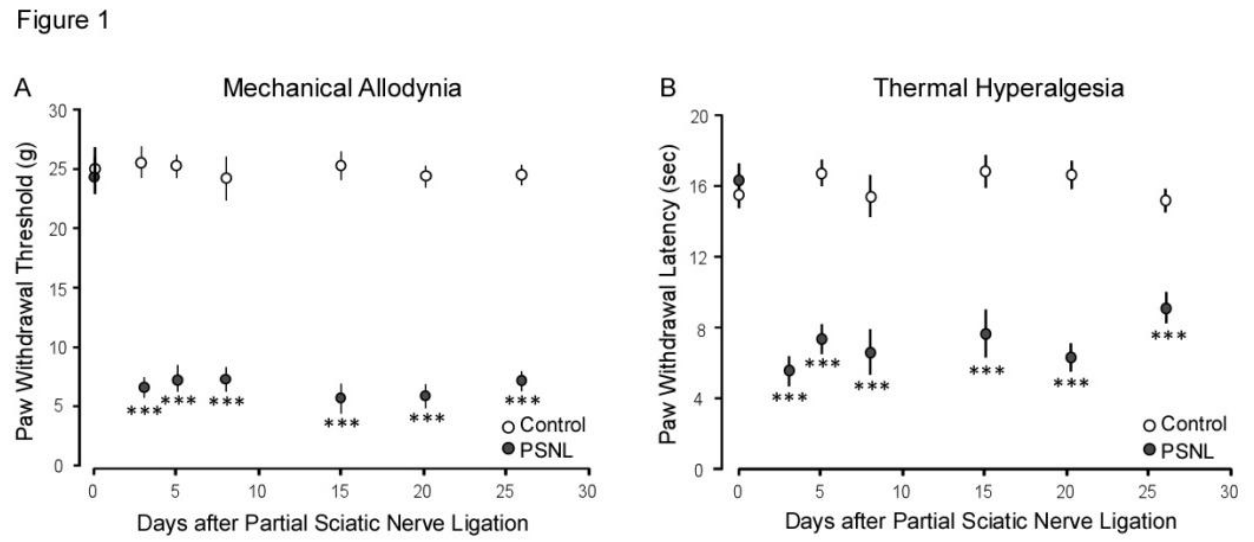

Figure1. PSNL operation results in stable neuropathic pain like behavior lasting at least 3 weeks. Rats in PSNL group showed reduced mechanical threshold (A) and thermal threshold (B). *** $\mathrm{P}<0.001$, vs. Control group, $n=6$ in Control group, $n=8$ in PSNL group.

\section{BoTN/A Intraplantar Injection Posterior to PSNL Operation}

The original intention of this study was to find the analgesic mechanism of BoTN/A for interpreting our clinical practice in which we used BoTN/A subcutaneous injection to treat postherpetic neuralgia. We gave a signal BoTN/A ipsilateral injection (i.pl.) to PSNL animals at day5 post operation after behavioral tests to mimic clinical treatment. The analgesic effect of BoTN/A could be observed from day2 post injection (PSNL day7) and maintained 3weeks (PSNL day 26) (Fig. 2).However, although the BoTN/A groups of both doses showed "statistical significance" comparing with PSNL group at the beginning, the curative effects presented quite limited and did not promote as time went on (Fig. 2).Dose dependence was also not observed. Even worse, serious side effects were concerned in high dose (40U/kg) group, including body weight loss and external hemorrhage at animals' canthi. 

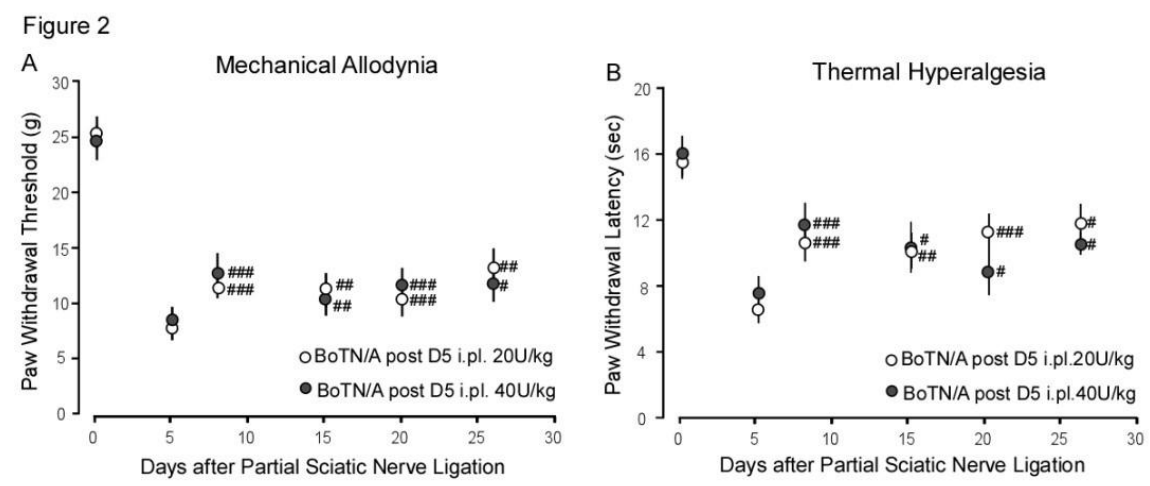

Figure2.Post-operation intraplantar injection of BoTN/A limitedly relieves PSNL induced mechanical allodynia and thermal hyperalgesia. At day 5 post operation, ipsilateral single BoTN/A i.pl. injection indeed made limited increase of both mechanical (A) and thermal (B) thresholds in PSNL animals without any dose dependence. \# $\mathrm{P}<0.05$, \#\# $\mathrm{P}<0.01$, \#\#\# $\mathrm{P}<0.001$, vs. corresponding PSNL group, $\mathrm{n}=5$ per group in BoTN/A i.pl. groups.

\section{BoTN/A Intraplantar Injection Previous to PSNL Operation}

To explore if BoTN/A could prevent the neuropathic pain, single BoTN/A was injected (i.pl.) into ipsilateral paw 3 days previous to PSNL operation (Fig. 3). The dose of $20 \mathrm{U} / \mathrm{kg}$ was chosen that all the experimental rats did not show any significant side effect. At the early phase, the BoTN/A group indeed revealed raised mechanical and thermal thresholds compared with corresponding PSNL group. However, these analgesic effects became weakened around 3weeks after PSNL operation. Even worse, at day26 after PSNL, mechanical allodynia in BoTN/A group was more significant than PSNL group that the rats showed lower mechanical threshold (Fig. 3A). The result indicated single BoTN/A pre-injection could only blocked PSNL induced neuropathic pain like behavior at the early phase.

Figure 3
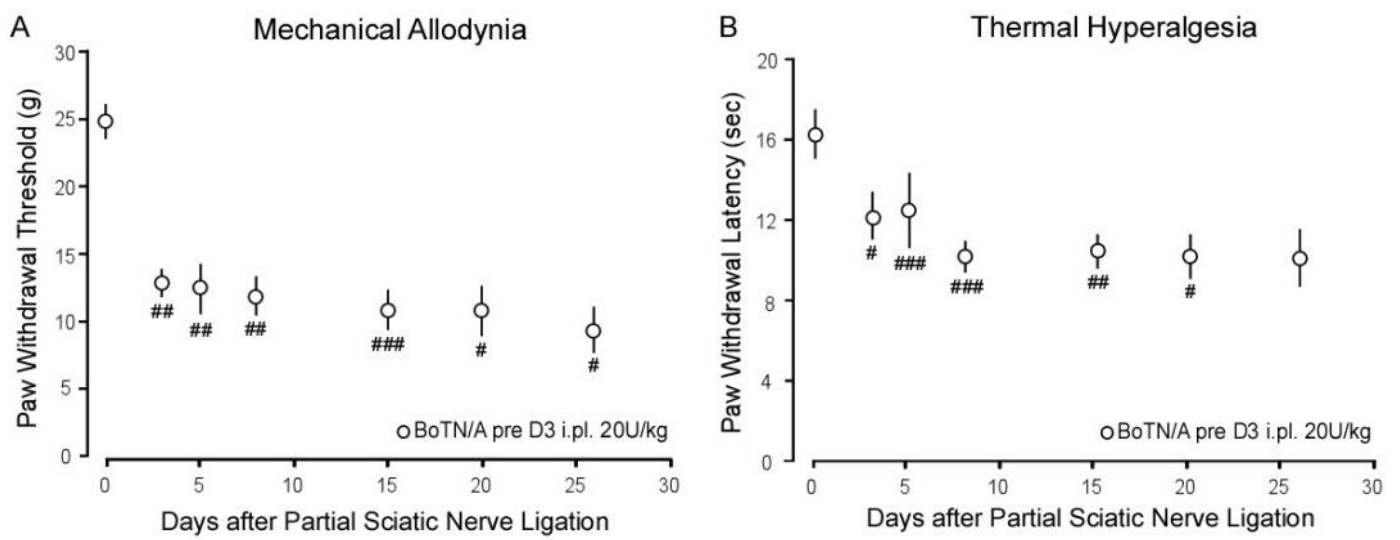

Figure3. Pre-operation intraplantar injection of BoTN/A limitedly relieves PSNL induced mechanical allodynia and thermal hyperalgesia at early stage. At 3 days previous to operation, single ipsilateral BoTN/Ai.pl. injection partially blocked PSNL induced mechanical allodynia and thermal hyperalgesia only lasting 3 weeks.\# $\mathrm{P}<0.05$, \#\# $\mathrm{P}<0.01$, \#\#\# $\mathrm{P}<0.001$, vs. corresponding $\mathrm{PSNL}$ group, $\mathrm{n}=5$ per group in BoTN/A i.pl. group.

\section{BoTN/A Intrathecal Injection Previous to PSNL Operation}

As previous research reported BoTN/A administered peripherally might be carried into central nervous system to make effect. As we all known, central hypersensitivity 
is an important factor for neuropathic pain initiation and maintenance, if BoTN/A could block PSNL induced central hypersensitivity, the neuropathic pain like behavior might be attenuated. Next, the direct central effect of BoTN/A was confirmed. Single high dose (1U/kg) BoTN/A injection (i.t.) 2 days previous operation relieved PSNL induced mechanical allodynia and thermal hyperalgesia to a great extent (Fig.4). The rats received high dose BoTN/A injection (i.t.) almost recovered at 4weeks after PSNL operation. However, in the low dose $(0.5 \mathrm{U} / \mathrm{kg})$ group, the same analgesic effect as the high dose group was not obtained, that the enlarged disparity of efficacy began to emerge at day 5 after PSNL operation. The intrathecal injection did not impact basic behavioral threshold. These date showed previous central administration of BoTN/A could positively prevent the initiation and maintenance of neuropathic pain.
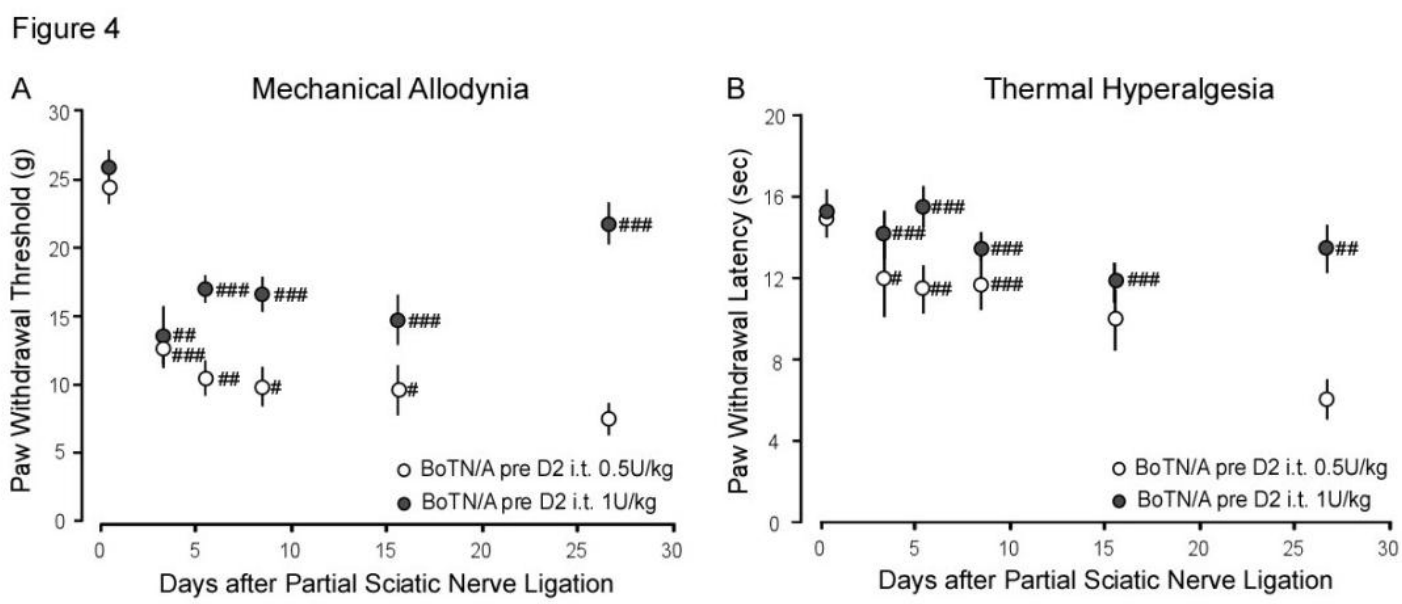

Figure4. Intrathecal injection of BoTN/A previous operation strongly attenuates PSNL induced thermal hyperalgesia from the early phase with significant dose dependent. At day 2 previous to operation, single BoTN/A i.t. injection significantly blocked PSNL induced mechanical allodynia(A) and thermal hyperalgesia(B). This analgesic effect showed distinct dose dependence that low dose $(0.5 \mathrm{U} / \mathrm{kg})$ maintained effect about 2 weeks. \# $\mathrm{P}<0.05$, \#\# $\mathrm{P}<0.01$, \#\#\# $\mathrm{P}<0.001$, vs. corresponding PSNL group, $\mathrm{n}=5$ per group in BoTN/A i.t. group.

\section{Discussion}

In this present study, we compared the analgesic effect of BoTN/A by different administration methods in PSNL induced neuropathic pain model. The experimental results demonstrate: 1)slight reduction of neuropathic pain is engendered by single intraplantar BoTN/Ainjection (20U/kg) at day 5 post operation; 2) high dose of BoTN/A (40U/kg) induce serious side effect; 3) pre-administration of BoTN/A at day 3 before operation by intraplantar injection is not better than post-administration; 4) pre-administration of BoTN/A at day 2 before operation by intrathecal injection with the relatively low dose $(1 \mathrm{U} / \mathrm{kg})$ shows long-lasting analgesic effect and benefits the animal recover from operation. It is suggested that central administration of BoTN/A is likely to be a novel approach to prevent operation induced neuropathic pain in the future.

The long lasting analgesic mechanisms of BoTN/A are considered by reducing pain related neurotransmitters and peptide release in peripheral and/or central nervous system; and reduction of local inflammation is also a factor which cannot be ignored[4]. The BoTN/A injected peripherally will be transported to central terminal to make bio-function, and the inhibition of retrograde axonal transport significantly compromises therapeutic efficacy of BoTN/A[12]. The sciatic nerve was ligation partly in our chosen model, thus the retrograde transport pathway was impeded 
ineluctably. Hence, the reason why do the BoTN/A intraplantar injection fail to obtain expected effect should be explained.

The most surprised discovery in this study is that previous intrathecal injection brings out favorable effect on blocking PSNL induced neuropathic pain. Moreover, the rats received intrathecal BoTN/A injection showed better recover than PSNL group. We have done research on the mechanism of this discovery and gotten some preliminary result (unpublished data). This discovery indicates BoTN/A is possibly used before the operation which could leave residual neuropathic pain in high probability. To enlarge the clinical application of BoTN/A, further studies are demanded.

\section{Summary}

The strong analgesic effect of BoTN/A by peripheral administration depends on axonal integrity, thus intraplantar injection does not display preconceived efficacy. Central administration of BoTN/A promises to be a preventative approach to neuropathic pain, especially for postoperative pain.

\section{Acknowledgements}

This study is supported by Shenzhen Committee of Technological Innovation (Project No.KQC201109050100A to Qiu Yunhai, Project No.JCYJ20140411091151446 to Xiao Lizu, and Project NoJC201105190910A to Xie Weijiao).

\section{References}

[1] Poulain, B. and Y. Humeau, Mode of action of botulinum neurotoxin: pathological, cellular and molecular aspect. Ann Readapt Med Phys, 2003. 46(6): p. 265-75.

[2] Dong, M., et al., SV2 is the protein receptor for botulinum neurotoxin A. Science, 2006. 312(5773): p. 592-6.

[3] Blasi, J., et al., Botulinum neurotoxin A selectively cleaves the synaptic protein SNAP-25. Nature, 1993. 365(6442): p. 160-3.

[4] Jabbari, B., Botulinum neurotoxins in the treatment of refractory pain. Nat Clin Pract Neurol, 2008. 4(12): p. 676-85.

[5] Attal, N., et al., EFNS guidelines on the pharmacological treatment of neuropathic pain: 2010 revision. Eur J Neurol, 2010. 17(9): p. 1113-e88.

[6] McMahon, H.T., et al., Tetanus toxin and botulinum toxins type A and B inhibit glutamate, gamma-aminobutyric acid, aspartate, and met-enkephalin release from synaptosomes. Clues to the locus of action. J Biol Chem, 1992. 267(30): p. 21338-43.

[7] Morris, J.L., P. Jobling, and I.L. Gibbins, Differential inhibition by botulinum neurotoxin A of cotransmitters released from autonomic vasodilator neurons. Am $\mathbf{J}$ Physiol Heart Circ Physiol, 2001. 281(5): p. H2124-32.

[8] Purkiss, J., et al., Capsaicin-stimulated release of substance P from cultured dorsal root ganglion neurons: involvement of two distinct mechanisms. Biochem Pharmacol, 2000. 59(11): p. 1403-6.

[9] Antonucci, F., et al., Long-distance retrograde effects of botulinum neurotoxin A. 
J Neurosci, 2008. 28(14): p. 3689-96.

[10] Xiao, L., et al., Subcutaneous injection of botulinum toxin a is beneficial in postherpetic neuralgia. Pain Med, 2010. 11(12): p. 1827-33.

[11] Shir, Y. and Z. Seltzer, Effects of sympathectomy in a model of causalgiform pain produced by partial sciatic nerve injury in rats. Pain, 1991. 45(3): p. 309-20.

[12] Bach-Rojecky, L. and Z. Lackovic, Central origin of the antinociceptive action of botulinum toxin type A. Pharmacol Biochem Behav, 2009. 94(2): p. 234-8. 